für Sexualdelikte etwa Albrecht (1999), Dessecker (1998) und Rosenau (1999: 394).

15 Statistisches Bundesamt (2004c: 148). Dabei sind zum Vergleich mit dem früheren Recht nur $\S 224$ I Nr. 2-5 StGB erfaßt.

16 Das ergibt sich aus einer Befragung des KFN in Niedersachsen und Schleswig-Holstein; siehe Schott et al. (2004: 356 ff.).

17 Dölling (2002: $61 \mathrm{ff}$.).

18 Zur langfristigen Entwicklung Jehle (2003: 27).

19 Ständige Rechtsprechung, z.B. BGH, Beschlüsse vom 14. Mai 1992 - 4 StR 178/92 (= StV 1993, 302) und zuletzt vom 29. Oktober 2002 - 3 StR 326/02 (= StraFo 2003, 100).

20 Ein laufendes Forschungsprojekt der KrimZ im Auftrag des Bundesministeriums der Justiz soll 2005 abgeschlossen werden. Bisher liegen erst unveröffentlichte Zwischenberichte vor; zuletzt Heimerdinger (2004).

21 Foth (1991); Maatz (1998); Rautenberg (1997); Streng (2003).

22 Siehe die zusammenfassende Entscheidung BGH, Urteil vom 17. Juni 2004 - 4 StR 54/04 (= HRRS 2004 Nr. 662).

23 BGH, Beschluß vom 27. Januar 2004 - 3 StR 479/03 (= NStZ 2004, 495).
24 Dafür spricht die jüngste Entscheidung BGH, Urteil vom 17. August 2004 - 5 StR 93/04 (= HRRS 2004 Nr. 847), die Bedenken gegen die vom Tatgericht angenommene Strafrahmenverschiebung äußert, aber zugleich eine Prüfung der Voraussetzungen des $\$ 64$ StGB empfiehlt.

25 BVerfG, Beschluß vom 16. März 1994 - 2 BvL 3/90 u.a. (= BVerfGE 91, 1, 28 ff.).

26Zum erstgenannten Gesichtspunkt Koller (2004), zum zweiten Schalast et al. (2005).

27 Juris-Abfrage am 15. Dezember 2004: Datum (1998-2004) und Normen (§ 67d Abs 2 StGB); N $=46$. Hinzu kommen zwei in Juris nicht erfaßte Revisionsentscheidungen des $\mathrm{BGH}$, die Hinweise zur Aussetzung einer Maßregel geben; siehe Beschlüsse vom 18. September 2000 - 5 StR 384/00 - und vom 18. Oktober 2000 - 1 StR 419/00 -, die beide in der elektronischen Datenbank Höchstrichterliche Rechtsprechung Strafrecht recherchiert werden können (http://www.hrr-strafrecht.de/hrr/bgh-db/index.php3).

28 KG, Beschluß vom 29. November 2001 - 5 Ws 646/01 (in Juris veröffentlicht) - 23 Jahre Freiheitsentziehung wegen Eigentumsdelikten.

29 OLG Koblenz, Beschluß vom 17. September 1998 - 1 Ws 172/98 (= NJW 1999, 876).
30 BVerfG, Beschluß vom 8. Oktober $1985-2$ BvR 1150/80 u.a. (= BVerfGE 70, 297, 315). Diese Rechtsprechung ist vom Bundesverfassungsgericht mehrfach bestätigt worden; siehe zuletzt BVerfG, Beschluß vom 4. Dezember 2003 - 2 BvR 1922/03 (= HRRS 2004 Nr. 72).

31 KG, Beschluß vom 31. März 1998 - 5 Ws $111 / 98$ (in Juris veröffentlicht).

32 Dessecker (1997: 117).

33 Seifert et al. (2001: 248).

34 Koller (2004).

35 Zum folgenden ausführlich Kröniger (2004: 24 ff.). 36 Dimmek (2003: 273); Seifert et al. (2001: 250).

37 Dessecker (1997: 121); Dimmek et al. (1996: 13); Jöckel \& Müller-Isberner (1994: 356); Seifert \& Leygraf (1997: 240 ff.).

38 Nedopil (2002: 211) mit Daten aus dem BKH Bayreuth. Im Niedersächsischen Landeskrankenhaus Moringen hat die durchschnittliche Unterbringungsdauer zum Stichtag 1. Juli von 3,9 Jahren 1996 auf 6,5 Jahre 2003 zugenommen (Spengler et al. 2003: 17). Weitgehend konstant seit 1997 ist die Unterbringungsdauer dagegen in der Hessischen Klinik für forensische Psychiatrie Haina (Müller-Isberner 2004). 39 So bereits die These von Koller (2004).

40 BGBl. I 1838.

41 Schneider (2004: 653 f.).

\title{
Führungsaufsicht am Scheideweg?
}

Frank Neubacher

\section{Einleitung}

ie Führungsaufsicht fristet, obwohl sie für die betroffenen Menschen und die Arbeit der sozialen Dienste der Justiz eine große Bedeutung hat, ein Schattendasein. Die Öffentlichkeit nimmt sie kaum wahr, und auch in den Kriminalwissenschaften beanspruchen andere Themen regelmäßig mehr Aufmerksamkeit als diese Maßregel der Besserung und Sicherung, die seit ihrer Einführung im Jahre 1975 wiederholt kritisch beurteilt worden ist. Die Strafrechtswissenschaft steht dem Institut der Führungsaufsicht überwiegend zwiespältig gegenüber ${ }^{1}$, und die Arbeitgemeinschaft Deutscher Bewährungshelfer forderte 1986 sogar seine völlige Abschaffung. Damit ist angesichts einer zunehmend auf Kontrolle und Sicherung bedachten Kriminalpolitik derzeit nicht zu rechnen. Vor dem Hintergrund eines ohnehin überlasteten Straf- und Maßregelvollzuges, dessen Probleme infolge der Ausweitung der Sicherungsverwahrung (s. § 66 bis $\S 66$ b StGB) nicht ab-, sondern zunehmen werden, unternimmt der folgende Beitrag den Versuch einer Bestandsaufnahme und Neuorientierung. ${ }^{2}$

\section{Rechtliche Grundlagen und kriminal- politische Positionen}

Die Führungsaufsicht verfolgt einen doppelten Zweck: Sie soll den Täter zum einen durch Überwachung und Kontrolle an der Begehung neuer Straftaten hindern (dient insoweit also dem Schutz der Allgemeinheit), sie soll ihn zum anderen aber auch durch Hilfe und Betreuung zu einem Leben ohne Straftaten befähigen. Der Gesetzgeber hat keiner der beiden Funktionen den Vorrang eingeräumt. Es ist nicht verwunderlich, dass dieses Spannungsverhältnis von Anfang an für die Vielzahl der auftretenden Probleme der Führungsaufsicht verantwortlich gemacht worden ist. Dieses Nebeneinander von Hilfe und Kontrolle kennen wir auch von der Strafaussetzung zur Bewährung, wo Gesichtspunkte des Schutzes der Allgemeinheit gleichfalls zu berücksichtigen sind. Zwei Unterschiede heben die Führungsaufsicht aber besonders von der Bewährungshilfe ab: Erstens beinhaltet die Führungsaufsicht eine umfassende Einwirkung auf den Täter, dem - anders als bei Bewährungsaussetzung - nicht nur bestimmte Weisungen und Auflagen erteilt worden sind. Und zweitens hat es die
Führungsaufsicht mit einer schwierigeren Klientel zu tun, weil die Unterstellung unter die Führungsaufsicht (anders als die Bewährungsaufsicht) keine positive Sozialprognose voraussetzt. ${ }^{3}$ Hinzu kommt, dass die betroffenen Verurteilten, denen der Unterschied zwischen Strafen einerseits und Maßregeln der Besserung und Sicherung andererseits nicht so leicht $\mathrm{zu}$ vermitteln sein dürfte, sich durch Überwachung und Kontrolle gegängelt fühlen schließlich haben sie ihre Strafen im Gegensatz zu Bewährungsprobanden vollständig verbüßt.

Drei Anwendungfälle der Führungsaufsicht sind zu unterscheiden:

1. Im Zusammenhang mit einer Freiheitsstrafe wird sie vom Tatrichter angeordnet bei einer Verurteilung $\mathrm{zu}$ einer Freiheitsstrafe wegen bestimmter, im Gesetz aufgeführter und als besonders rückfallträchtig eingeschätzter Straftaten (s. § 68 Abs. 1 StGB). Solche Taten sind im Strafgesetzbuch sowie im Nebenstrafrecht $\mathrm{zu}$ finden (Beispiele: bestimmte Sexualstraftaten, § 181b StGB, erpresserischer Menschenraub und Geiselnahme, § 239c StGB, Diebstahl, § 245 StGB, Raub und Erpressung, § 256 Abs. 1 StGB, Hehlerei und Geldwäsche, § 262 StGB, Betrug, § 263 Abs. 6 StGB, sowie bestimmte Verstöße gegen das Betäubungsmittelgesetz, § 34 BtMG).

2. Kraft Gesetzes tritt sie ein, wenn der Täter wegen einer vorsätzlichen Straftat zu einer Freiheitsstrafe von mindestens zwei Jahren oder wegen einer Sexualstraftat zu einer Freiheitsstrafe von mindestens einem Jahr verur- 
teilt worden ist und die Strafe vollständig verbüßt hat (§ 68f Abs. 1 StGB); nach überwiegender Auffassung kann das Mindestmaß auch durch eine Gesamtstrafe erfüllt werden.

3. Und drittens ist die Führungsaufsicht im Zusammenhang mit freiheitsentziehenden Maßregeln gesetzlich vorgesehen, wenn die Vollstreckung der Maßregel zur Bewährung ausgesetzt wird (s. v. a. § 67b Abs. 2 StGB, § 67d Abs. 2 Satz 2 StGB), wenn das Gericht die Unterbringung in der Sicherungsverwahrung nach Ablauf von zehn Jahren für erledigt erklärt ( $\$ 67 \mathrm{~d}$ Abs. 3 Satz 2 StGB) oder wenn die Unterbringung in einer Entziehungsanstalt erfolgt ist und der Maßregelzweck aus Gründen, die in der Person des Täters liegen, nicht erreicht werden kann (§ 67d Abs. 5 Satz 2 StGB).

Aufgrund der Vielzahl der Anwendungsfälle haben es die Führungsaufsichtsstellen mit einer sehr heterogenen Klientel zu tun, die von schuldfähigen »Vollverbüßern« über Suchtmittelabhängige bis zu ehemaligen Psychiatriepatienten reicht, die sich bewähren sollen. Heterogen sind folgerichtig auch die Risikoprognosen und Problemlagen, so dass die Führungsaufsicht vor der Aufgabe steht, ihre beschränkten Ressourcen differenziert und zielgruppengenau einzusetzen. ${ }^{4}$ Diese Aufgabe ist nicht einfacher geworden, seitdem der Gesetzgeber im Zusammenhang mit dem Gesetz zur Bekämpfung von Sexualstraftaten und anderen gefährlichen Taten 1998 die Schwelle für die Verhängung der Sicherungsverwahrung abgesenkt und die zeitliche Dauer, für die Führungsaufsicht angeordnet werden kann, erhöht hat (s. \$§ 68c Abs. 2, 68e Abs. 4 $\mathrm{StGB}$ ). Dabei ist die Führungsaufsicht schon lange im Gerede. Der Streit ist von Anfang an grundsätzlicher Natur gewesen. ${ }^{5}$ Die vertretenen Positionen reichen von der Ausweitung der Führungsaufsicht, etwa durch eine Absenkung der MindestverbüBungszeiten bei $\S 68 \mathrm{f} \mathrm{Abs.} 1$ StGB, bis hin zu ihrer ersatzlosen Streichung. Dazwischen liegt der Vorschlag, die Führungsaufsicht durch eine automatische Reststrafenaussetzung mit Bewährungsunterstellung zu ersetzen. Kritisiert werden insbesondere der Zielkonflikt der Führungsaufsicht, also die Vermischung von Hilfe und Kontrolle, die Doppelbetreuung von Unterstellten durch die Bewährungshilfe und Führungsaufsichtsstellen, der heterogene Personenkreis der unter Führungsaufsicht gestellten Menschen ${ }^{6}$ sowie die zunehmende Ressourcenknappheit bei Bewährungshilfe und Führungsaufsicht.

Für eine Reform der Führungsaufsicht stehen derzeit zwei Modelle zur Diskussion - vorausgesetzt natürlich, man möchte sie weder so belassen, wie sie jetzt ist, noch in Gänze abschaffen. Erstens ließe sich die Führungsaufsicht ersetzen, wenn man ein Modell der automatischen Vollstreckungsaussetzung mit Bewährungsunterstellung einführte. Die Reststrafenaussetzung wäre dann nicht mehr von einer gesonderten Prognoseentscheidung der Strafvollstreckungskammer abhängig, die Probanden könnten komplett von der klassischen Bewährungshilfe übernommen werden und der verbleibende Strafrest könnte als »Druckmittel « eingesetzt werden, um die Kooperation des Unterstellten herbeizuführen. Schwachpunkte dieser Lösung bestehen darin, dass ein Verurteilter dann auch gegen seinen Willen aus der Haft entlassen werden müsste, was mit der gegenwärtigen Rechtslage nach § 57 StGB nicht in Einklang zu bringen ist und auch verfassungsrechtliche Fragen im Hinblick auf den Rechtsanspruch auf Behandlung und Resozialisierung aufwirft. Während mir diese Punkte durchaus lösbar erscheinen (etwa durch eine Behandlung in Freiheit), begegnet dieses Modell in anderer Hinsicht Bedenken. Offensichtlich geht es nämlich davon aus, dass sich die Klientel der Führungsaufsicht nicht wesentlich von der der Bewährungshilfe unterscheide und deshalb von dieser übernommen werden könnte. Hier setzen aber Bedenken an. Eine schlechte Sozialprognose kann zwar durch einen Federstrich des Gesetzgebers (im Rahmen des Ersetzungsmodells) für unbeachtlich erklärt werden, die tatsächlichen Probleme dieser Personen bleiben aber natürlich weiter virulent.

So wie im Strafvollzug die Gruppen der Sexual- und Gewaltstraftäter sowie der drogenabhängigen Täter seit Jahren anwachsen, so wird auch von Führungsaufsichtsstellen berichtet, sie hätten im Gegensatz zur Bewährungshilfe, die schwerpunktmäßig immer noch mit Eigentumsdelikten zu tun habe, eine besonders problematische Klientel mit langjähriger Kriminalitätserfahrung und erheblichen Vorstrafen zu versorgen, die vor allem aus dem Bereich der Sexualund Gewaltdelikte stamme. Wenn aber die Prämisse einer vergleichbaren Klientel nicht stimmt, dann böte sich statt des Ersetzungmodells eine andere Reformlösung an, die ich »Entflechtungsmodell« nennen möchte. Ihr Ziel wäre die Entlastung der Führungsaufsichtsstellen von Probanden mit geringerem Risikopotential und die Konzentration und Spezialisierung auf besonders belastete Personen. Wie eine solche Fokussierung unter den Vorzeichen einer zunehmend an Sicherungsaspekten ausgerichteten Kriminalpolitik aussehen könnte, hat der Gesetzgeber (immerhin!) vorgemacht, als er 2002 in $§ 66 \mathrm{a}$ StGB die vorbehaltene Sicherungsverwahrung eingeführt hat, sie aber auf solche erheblichen Straftaten begrenzte, durch welche die Opfer seelisch oder körperlich schwer geschädigt werden (§ 66 Abs. 2 Satz 2 StGB). Diebstahl und Betrug können damit kaum gemeint sein. Über eine solche deliktsgebundene Limitierung der Führungsaufsicht hinaus könnte weiterer Spielraum für die Arbeit der Führungsaufsichtsstellen geschaffen werden, wenn man die Führungsaufsicht generell und zusätzlich - auf die sog. Vollverbüßer (§ 68f StGB), auf wegen Therapieunfähigkeit aus einer Entziehungsanstalt Entlassene $(\S 67 \mathrm{~d}$ Abs. 5 StGB) sowie auf jene beschränkte, die nach zehn Jahren aus der Sicherungsverwahrung entlassen werden ( $(67 \mathrm{~d}$ Abs. 3 StGB). Teil dieser Strategie wäre die Verhinderung der weiteren ge- setzlichen Ausdehnung der Führungsaufsicht (etwa durch Absenkung der Mindestverbüßungsdauer). Überdies ließe sich das bisherige Nebeneinander von Bewährungshilfe und Führungsaufsicht reduzieren.

\section{Kriminalstatistische und empirische Befunde}

Kaum eine Zahl vermag die Bedeutung der Bewährungshilfe für die Rechtspflege in Deutschland und ihr Sanktionensystem besser zu veranschaulichen als die Relation zwischen Strafgefangenen und den Unterstellungen unter Bewährungsaufsicht. Im Jahre 1997 gab es im gesamten Bundesgebiet 51.442 Strafgefangene (2002: 60.443$)^{7}$, aber 141.195 Unterstellungen unter Bewährungsaufsicht allein im früheren Bundesgebiet einschließlich Berlin-Ost (ohne Hamburg). Dabei ist zu berücksichtigen, dass einzelne Probanden auch mehrfach unter Bewährung stehen können, die Zahl der Bewährungsprobanden also etwas niedriger liegt. Auf der anderen Seite bezieht sich die genannte Zahl an Bewährungsunterstellungen nur auf das frühere Bundesgebiet. Auf dieser Basis lässt sich also vorsichtig schätzen, dass auf einen Strafgefangenen wenigstens drei Personen unter Bewährungsaufsicht kommen. In groben Zügen finden wir in diesen Zahlen die Sanktionsstruktur widergespiegelt. Bekanntlich entfallen von 100 strafrechtlichen Verurteilungen rund 80 auf eine Geldstrafe, 15 auf eine Bewährungsstrafe und nur 5 auf eine vollstreckbare Freiheitsstrafe.

In diesen Zahlen sind noch nicht enthalten die Unterstellungen unter Führungsaufsicht. Daten sind insoweit nicht verfügbar, weil die Führungsaufsichtsstatistik auf Beschluss der Länder in den neunziger Jahren eingestellt wurde und die Bewährungshilfestatistik die Führungsaufsicht nicht mehr ausweist. Aus der Strafverfolgungsstatistik lassen sich wiederum nur die richterlich angeordneten Führungsaufsichten ersehen. Demzufolge wurden im Jahr 2001 durch die deutsche Justiz gegen 46 Erwachsene, 14 Heranwachsende und 10 Jugendliche Führungsaufsicht angeordnet. In der Summe sind das 70 richterlich angeordnete Unterstellungen unter Führungsaufsicht. ${ }^{8}$ Das ist bekanntlich nur ein verschwindender Bruchteil der tatsächlich unter Führungsaufsicht stehenden Personen; denn die Fälle, in denen die Führungsaufsicht kraft Gesetzes eintritt, sind sehr viel zahlreicher. Schöch hat für das Jahr 1989 insgesamt 12.400 Unterstellungen unter Führungsaufsicht ermittelt. Der Anteil der Führungsaufsichten an allen Unterstellungen betrug damals 8,7 \%. In absoluten Zahlen blieb die Unterstellung unter Führungsaufsicht seit 1975 lange relativ konstant. Wie sie sich in den letzten Jahren entwickelt hat, wissen wir nicht genau; vieles spricht aber für einen rasanten Anstieg. Für ganz Deutschland wird die Zahl der Führungsaufsichtsfälle auf derzeit ca. 20.000 geschätzt. ${ }^{9}$ Indizien für einen starken Anstieg sind der Umstand, dass allein in Nordrhein- 
Westfalen im Jahre 2002 mehr als 4.100 Personen unter Führungsaufsicht standen ${ }^{10}$, sowie die Steigerung der Führungsaufsichtsfälle in Bayern um $56 \%$ - allein in den letzten fünf Jahren. ${ }^{11}$ Auch Praktiker und Praktikerinnen aus Bewährungshilfeund Führungsaufsichtsstellen haben den Eindruck, die Zahl der Unterstellungen unter Führungsaufsicht nehme dramatisch zu. Wenn man die Entwicklung der letzten Jahrzehnte unter einem dynamischen Aspekt betrachet, dann wird deutlich, dass Führungsaufsicht und Bewährungshilfe nicht nur quantitativ belastet sind, weil die Unterstellungen unter Bewährungshilfe in diesem Zeitraum stark zugenommen haben. Auch qualitativ werden sowohl die Führungsaufsicht als auch die Bewährungshilfe stark gefordert; denn die Zurückdrängung stationärer Maßnahmen durch die Ausweitung der Strafaussetzung hat in den letzten Jahrzehnten dazu geführt, dass »heute im Vergleich zu den 60er Jahren wesentlich mehr Problemprobanden mit erheblichen Sozialisationsdefiziten zu betreuen « sind. ${ }^{12}$ Da sich in diesem Zeitraum aber (wie im übrigen auch im Strafvollzugi3) die Betreuungsrelation, also die Zahl der zu Betreuenden pro Bewährungshelfer, eher verschlechtert hat, ist die reservierte Haltung vieler Bewährungshelfer gegenüber der Führungsaufsicht zumindest nachvollziehbar. Sie sind mit etwa 70 Bewährungsprobanden in der Regel schon voll ausgelastet. ${ }^{14}$

Vorhandene empirische Befunde dämpfen auch die Erwartung, die Führungsaufsichtsstellen könnten unter den gegebenen Umständen in nennenswertem Umfang Hilfestellung bieten. Brusten $^{15}$ bemängelte schon 1983, dass sich die Führungsaufsicht auf den Kontrollaspekt konzentriere, weniger auf Hilfe. Und Kurze zählte durchschnittlich 171 Fälle, die ein in der Führungsaufsicht Tätiger zu bewältigen hat. Bei diesem Arbeitsaufkommen versteht es sich von selbst, dass eine intensive Betreuung nicht möglich ist. In der täglichen Praxis kommt es zu einem Arrangement in der Arbeit mit den Probanden. Die Rollen sind in der Regel so verteilt, dass die Führungsaufsichtsstelle den Erstkontakt mit dem Probanden herstellt und überwiegend die Überwachungsund Verwaltungsaufgaben wahrnimmt, während sich die Bewährungshilfe auf Hilfs- und Betreuungsmaßnahmen konzentrieren soll. Scheinbar werden durch diese Rollenverteilung in der Praxis Reibungsverluste überwiegend vermieden. Jedenfalls berichten die meisten in der Führungsaufsicht tätigen Sozialarbeiter von einem guten Verhältnis zur Bewährungshilfe, während die Kooperation mit anderen Stellen (z.B. psychiatrische Anstalten, Entziehungsanstalten, Polizei, Gerichtshilfe) als problematischer eingeschätzt wird. Die im Schrifttum gelegentlich anzutreffende Charakterisierung der Führungsaufsicht als »Aktendurchlaufstelle» wird in der Untersuchung eher bestätigt als widerlegt. Die meisten Befragten beschränkten sich auf einige wenige telefonische Kontakte und Ladungen; Hausbesuche sind im Gegensatz zur Bewährungshilfe selten. ${ }^{16}$ Im Übrigen zeigte Kurzes Untersuchung erneut, dass die
»Vollverbüßer« weit mehr als die Hälfte der Klientel der Führungsaufsicht ausmachen und damit der Kontrollaspekt stark im Vordergrund steht.

\section{Thesen zur Zukunft der Führungs- aufsicht}

Wie sich die derzeitige kriminalpolitische Situation auf die Führungsaufsicht auswirken wird, ist schwer absehbar. Der Strafvollzug hat nach wie vor mit Phänomenen der Überbelegung $\mathrm{zu}$ kämpfen - im Jahre 2002 erreichte die Zahl der inhaftierten Strafgefangenen mit 60.443 einen Höchststand. Aus diesem Grunde ist zu besorgen, dass eine zunehmende Zahl von »Vollverbüßern«, denen zuvor eine Reststrafenaussetzung verwehrt worden ist, künftig die Führungsaufsichtsstellen noch weiter belasten.

Das Bundesverfassungsgericht ${ }^{17}$ hat mit seinem Beschluss aus dem Jahre 1980 entschieden, auch die nach $\S 68 \mathrm{f}$ StGB kraft Gesetzes eintretende Führungsaufsicht begegne keinen verfassungsrechtlichen Bedenken. Es handele sich dabei nämlich nicht um eine unzulässige erneute Bestrafung, sondern um eine an die ursprüngliche Verurteilung angeschlossene Maßregel der Besserung und Sicherung, deren Wirksamwerden lediglich von der vollständigen Verbüßung der Freiheitsstrafe abhänge. Derartige Nebenfolgen der Verurteilung seien, ohne dass es eines richterlichen Ausspruches bedürfe, für den Betroffenen ohne Weiteres erkennbar. Zwar bleibe der Verurteilte bis zur Entscheidung über die Aussetzung des Strafrestes, unter Umständen sogar bis zum Ende der Strafverbüßung, im Unklaren, ob die Führungsaufsicht tatsächlich eintrete. Das widerspreche aber nicht den vom Rechtsstaatsgebot an die Voraussehbarkeit gesetzlicher Maßnahmen geknüpften Anforderungen. Wegen der negativen Sozialprognose verstoße der Eintritt der Führungsaufsicht nicht gegen den verfassungsrechtlichen Grundsatz der Verhältnismäßigkeit, und auch das Grundrecht der Berufsfreiheit werde durch eine Weisung, einer vom Bewährungshelfer gebilligten, versicherungspflichtigen Arbeit nachzugehen, nicht verletzt. Das Rechtsstaatsprinzip verbiete - so die Schlussfolgerung des Bundesverfassungsgerichts - lediglich belastende Gesetze, die zur Erreichung der Gesetzeszwecke schlechthin untauglich seien; davon könne hier aber nicht die Rede sein.

Ob das Gericht die Frage der Tauglichkeit der Führungsaufsicht heute in Kenntnis der empirischen Befunde unter verfassungsrechtlichen Aspekten anders beurteilen würde, ist zu bezweifeln. Von einem kriminalpolitischen Standpunkt aus möchte ich diese Frage aber thesenartig aufgreifen: Zur Zeit geht die kriminalpolitische Entwicklung dahin, strafrechtliche Sozialkontrolle unter Betonung von Sicherheit, Sicherheitsgefühl und Prävention auszubauen. Die Diskussion um die sog. nachträgliche Sicherungsverwahrung belegt das. Mit einer Abschaffung des Instituts der Führungsaufsicht ist daher ge- wiss nicht zu rechnen. Auf der anderen Seite kann es jedoch schwerlich so weitergehen wie bisher. Eine Reform der Führungsaufsicht ist nötig, weil sie ihrer ursprünglichen Intention, Kontrolle und Hilfe zu leisten, nicht mehr gerecht wird. Angesichts steigender Unterstellungen (bei Bewährungshilfe und Führungsaufsicht), angesichts der Überlegungen, die Führungsaufsicht auszuweiten durch eine Reduzierung der Mindestverbüßungsdauer der verhängten Freiheitsstrafe, und angesichts knapper werdender personeller Ressourcen, die die Betreuungsrelation weiter verschlechtern werden, bleibt die Hilfestellung durch die Führungsaufsicht ein leeres Versprechen. In dieser Situation könnte die Führungsaufsicht vielleicht Schlimmeres verhindern, wenn das Gesetz den in der Praxis ohnehin dominierenden Kontroll- und Überwachungsaspekt betonte und die Führungsaufsicht im Gegenzug unter Freistellung von anderen Aufgaben in die Lage versetzte, Sicherungsverwahrung so weit wie möglich durch Kontrolle in Freiheit zu ersetzen.

PD Dr. iur. Frank Neubacher M.A., Institut für Kriminologie, Köln

\section{Fußnoten:}

1 Vgl. Schönke/Schröder-Stree, StGB, 26. Aufl 2001, § 68 Rn. 2; Tröndle/Fischer, StGB, 51. Aufl. 2003, Vor § 68 Rn. 1; Lackner/Kühl, StGB, StGB, 24. Aufl. 2001, Vor $\$ 68$ Rn. 1.

2 Vgl. Neubacher, Führungsaufsicht, quo vadis? Eine Maßregel zwischen Sozialkontrolle und Hilfsangebot, in: BewHi 2004, S. 73-84.

3 Vgl. Meier, Strafrechtliche Sanktionen, 2001, S. 240; Streng, Strafrechtliche Sanktionen, 2. Aufl. 2002, S. 171.

4 Meier, Strafrechtliche Sanktionen, 2001, S. 243.

5 Vgl. etwa Dertinger/Marks (Hrsg.), Führungsaufsicht, 1990; Dünkel/Spiess, BewHi 1992, S. 129; Lemke/Vetter, BewHi 1992, S. 147 ff.; Antons, BewHi 1992, S. 282 ff.

6 Vgl. Lemke/Vetter, BewHi 1992, S. 147

7 Einschließlich Sicherungsverwahrung und Jugendstrafe, ohne U-Haft, Zahlen jeweils zum Stichtag 31.3., s. Statistisches Bundesamt, Rechtspflege, Fachserie 10, Reihe 4.1.

8 Statistisches Bundesamt, Rechtspflege, Fachserie 10, Reihe 3, Tab. 5.4, S. 77.

9 Vgl. Schöch, BewHi 2003, S. 224. Mitteilung aus dem Justizministerium Nordrhein-Westfalen. 10 Schöch, BewHi 2003, S. 214.

11 Schöch, NStZ 1992, S. 366.

$12 \mathrm{Vgl}$. Hohage/Walter/Neubacher, Die Entwicklung der personellen Ausstattung der Justizvollzugsanstalten in Abhängigkeit von kriminalpolitischen Strömungen, in: Zeitschrift für Strafvollzug und Straffälligenhilfe 2000, S. 136 ff. - gemeint ist das Verhältnis der Fachdienste (Sozialarbeiter, Psychologen u.ä.) zu den Strafgefangenen.

13 Vgl. Schöch, NStZ 1992, S. 367.

14 Brusten, Zwischen Hilfe und Kontrolle, Eine empirische Untersuchung zur Struktur und Entwicklung der Probanden der Führungsaufsicht, in: Kerner/Kury/Sessar (Hrsg.): Deutsche Forschungen zur Kriminalitätsentstehung und Kriminalitätskontrolle, German Research on Crime and Crime Control, Band 6/3, 1983, S. $1613 \mathrm{ff}$.

15 Vgl. Kurze, Soziale Arbeit und Strafjustiz, 1999 S. 466.

16 BVerfGE 55, $28 \mathrm{ff}$. 